\title{
MOBILE PHONE USERS AND ITS EFFECT OF HEARING IN TERMS OF DISTORTION PRODUCT OTOACOUSTIC EMISSION (DPOAE)
}

\author{
Gaurav Kumar1, Nitin Gupta², Nirmal Kumar Sinha ${ }^{3}$ \\ ${ }^{1}$ Assistant Professor, Department of ENT, T. S. Misra Medical College, Lucknow, Uttar Pradesh, India. \\ ${ }^{2}$ Senior Resident, Department of ENT, T. S. Misra Medical College, Lucknow, Uttar Pradesh, India. \\ ${ }_{3}^{3}$ Audiologist, Department of ENT, T. S. Misra Medical College, Lucknow, Uttar Pradesh, India.
}

\begin{abstract}
\section{BACKGROUND}

Cellular phones/ mobile phones are the new addiction in today's society. They can do pretty much everything from making calls to controlling remotely located devices/cameras. Mobile phones, owing to their user-friendly interface and reasonable prices are being used by people from every socioeconomic section and almost all the age groups. Such a rampant use of these devices makes them potentially powerful tools. The convenience, that these devices offer, easily overlooks the cost our health has to pay because of their continuous and prolonged use. The main technology that these devices use nowadays is Global System for Mobile Communications (GSM). Mobile phones emit a pulsed high-frequency electromagnetic (EM) field that may have adverse effects. The Disturbances of the mechanoelectrical transduction during cellular phone use, especially because there is a close proximity of the cellular phone to the cochlea resulting in a fairly high absorption rate of the incident electromagnetic field (EM).
\end{abstract}

Aim- To assess hearing loss in mobile phone users.

\section{MATERIALS AND METHODS}

This is a descriptive study conducted among mobile phone users in the Department of ENT at T.S. Misra Medical College and Hospital from January 2017 to June 2018 consisting of 190 healthy volunteers between the age of 21 and 50 years.

\section{RESULTS}

Mostly in Rt. handed mobile user had shown Refer/Fail OAE 21\%, out of which, higher frequency $6 \mathrm{KHz}$ is affected more in comparison of all four frequency of $(2,3,4 \& 6 \mathrm{KHz})$.

\section{CONCLUSION}

The study concludes that the use of mobile phones can cause damage to the Outer Hair Cells (OHCs) in the Cochlea especially in the basal turn which may impair hearing in long term use. Oto Acoustic Emissions from the cochlea can reliably detect this damage and thus has proven to be an excellent diagnostic tool.

\section{KEY WORDS}

Mobile Phone, Electromagnetic Field, DPOAE.

HOW TO CITE THIS ARTICLE: Kumar G, Gupta N, Sinha NK. Mobile phone users and its effect of hearing in terms of distortion product otoacoustic emission (DPOAE). J. Evolution Med. Dent. Sci. 2018;7(52):5520-5523, DOI: 10.14260/jemds/2018/1222

\section{BACKGROUND}

Cell phones, mobile phones, smart phones are the many names of a device around which our present lives revolve. Frequently, they are the first things we see when we get up in the morning, to check any missed calls, messages or multimedia notifications. More often they are the last things we see before going to sleep when we set an alarm for the next morning. And in between we intermittently use them to answer/make phone calls, to send/receive instant messages and for numerous other tasks which otherwise would take a longer time. But having a phone makes it all so easy and convenient. By just being at a place we can do just about anything which would have been a dream in the days bygone.

'Financial or Other Competing Interest': None.

Submission 06-11-2018, Peer Review 11-12-2018,

Acceptance 17-12-2018, Published 24-12-2018.

Corresponding Author:

Gaurav Kumar,

1404, HIG, Sector-I,

L. D. A, Kanpur Road,

Near Pakri Pul,

Lucknow-226012,

Uttar Pradesh, India.

E-mail: kumargaurav.1014@gmail.com

DOI: $10.14260 /$ jemds/2018/1222

\section{(c) $($ ) $\$$}

Everyone, I mean everyone uses/ or has used a mobile phone in his lifetime. Its contribution/participation in our daily/personal life has never been more invasive and ingrained. Smart phones, owing to their user-friendly interface and reasonable prices are being used by people from every socioeconomic section and almost all the age groups. Even small toddlers barely mumbling monosyllables can very much stare at the smart phones screens if it attracts them. Children use it for communication, playing games, watching videos and surf the internet. Young teenagers use social media applications like Facebook, Snapchat, WhatsApp, and Twitter to socialize and communicate. Features like email, scanner, job search engines, real estate selling and buying apps, Doctor's blogs and an endless array of other office applications make them useful to the professionals. Even the retired old people use these devices to talk and communicate with their children, grandchildren and their friends. Such a rampant use of these devices makes them potentially powerful tools. The convenience, that these devices offer, easily overlooks the cost our health has to pay because of their continuous and prolonged use. Global System for Mobile Communications (GSM) is the commonest international operating standard for the second generation of digital cellular mobile communications. It is used in Europe, Africa and Asia. It enables mobile phones to be used across 
national boundaries. This technology operates in the 900 $\mathrm{MHz}$ and $1800 \mathrm{MHz}$ frequency bands. CDMA is another technology that allows for the highest capacity and highest mobile data throughput. Proponents of Code Division Multiple Access (CDMA) also claim that there are fewer dropped calls when crossing cell boundaries.[1] CDMA is not commonly used in India.

Mobile phones emit a pulsed high-frequency electromagnetic (EM) field that may have adverse effects. Mobile phone networks operate in one of three bands; 900 $\mathrm{MHz}, 1800 \mathrm{MHz}$ and $2.2 \mathrm{GHz}$. The rapid adoption of wireless communication, especially handheld cellular phones, has generated controversy over whether or not these systems with electromagnetic fields (EMFs) pose a potential risk to human health. They transmit and receive microwave radiation at frequencies mainly ranged between 800 and $2000 \mathrm{MHz}^{[2]}$ which excites rotation of water molecules and some organic molecules but is non-ionizing [3] and so would not be expected to damage DNA. This microwave radiation has been reported to have thermal and non-thermal effects in humans. ${ }^{[4]}$ Headaches, sensations of burning or warmth of the ear, burning sensations in the facial skin and alteration of the blood-brain barrier are the reported thermal effects resulting from mobile telephone use. ${ }^{[5,6]}$ Non-thermal effects described in the literature include modifications of sleep patterns (detected by means of electroencephalography), an increase in blood pressure and effects on cognitive function. $[7,8,9]$

The International Commission on Non-Ionizing Radiation Protection (ICNIRP) ${ }^{[10]}$ formulates and publishes exposure limit guidelines for electromagnetic (EM) field radiation, based on critical review of the published biological effects and health risks. In the frequency range $10 \mathrm{MHz}$ to $10 \mathrm{GHz}$, this encompasses mobile phone related electromagnetic (EM) radiation; near-field models of energy absorption become important. Local (That is anatomical regional) energy absorption can be high and energy density reported as specific absorption rate in units of watts/kilograms specific absorption rate (SAR) is the recommended dosimetric measure of the same. Of all the anatomical structures, the ear is closest to the mobile phone,[11,12] which may lead to relatively high energy exposure of the ear compared to other parts of the body. The fluid-filled inner ear possesses micro homeostatic mechanisms that are essential for the functioning of the mechanoelectrical transduction of the auditory hair cells in the cochlea. Thus, disturbances of the mechanoelectrical transduction and changes due to thermal effects may appear during cellular phone use, especially because there is a close proximity of the cellular phone to the cochlea resulting in a fairly high absorption rate of the incident electromagnetic field (EMF). Exposure standards set by national and international institutions, limit the values for specific absorption rate (SAR) of 1.6 to $2 \mathrm{~W} / \mathrm{kg}$ for local exposure to the general public. [13]

\section{Aims and Objectives}

To assess the hearing loss in mobile phone users.

\section{MATERIALS AND METHODS}

This is a descriptive study among mobile phone users was conducted in the Department of ENT at T.S. Misra Medical College and Hospital, Lucknow over a period of one and a half years from January 2017 to June 2018. The study group consisted of 190 healthy volunteers between the age group of 21 and 50 years. The candidates with other known causes of hearing loss like ototoxic drug use, diabetes, those working in noisy environments like explosives, firearms, industrial machines, pneumatic drills, sirens, recurrent/ recent ear, nose complaints, those with history/ complaints of ear discharge were excluded from the study after screening via history taking and ENT examination. The finally selected candidates underwent detailed history taking regarding hand dominance, duration, pattern and years of mobile use and then underwent thorough clinical examination before being subjected to DPOAE. All candidates were subjected to distortion product otoacoustic emission (DPOAE) test in both the ears.

\section{RESULTS}

We included 190 candidates in the study who fulfilled the criteria. Among the 190 subjects, 112 were males and 78 were females. Male - Female ratio is $59 \%$ and $41 \%$ respectively (Fig. 1). The age of the volunteers was between 21 and 55 years with mean age of 31 years.

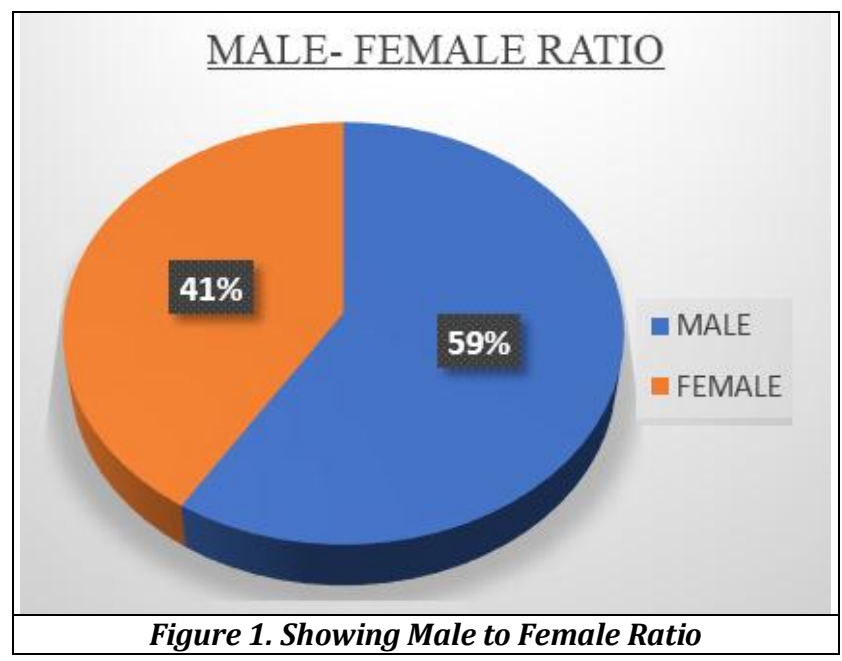

The subjects were then categorized according to the handling of mobile phone in either Right Handed or Left handed. Total of 190 subjects, 149 were Right handed and 41 were Left handed while using mobile phones while 56 preferred using either hands.

Out of 149 people who use Right hand for answering the phone, 111 people strictly use Right Hand while 38 people either use Right or Left hand for answering the calls, (Fig 2). Out of 41 people who use Left hand for answering the phone, 23 people strictly use Left Hand while 18 people either use Right or Left hand for answering the calls.

We ask about the associated complaints using the mobile phone out of 190 subjects, 33 people have complained of heaviness or blocked sensation in ears, 28 people have Tinnitus, 15 people have hearing loss and 151 people have no complaints.

DPOAE measurements were made on both side of Ear. In Right side it is Pass/ Present in 151 people (79\%) and Refer/Absent in 39 people (21\%) had OAE absent, while on left side 160 people (84\%) had OAE Pass/ Present and 30 people (16\%) had Refer/Absent OAE, (Fig. 2). 


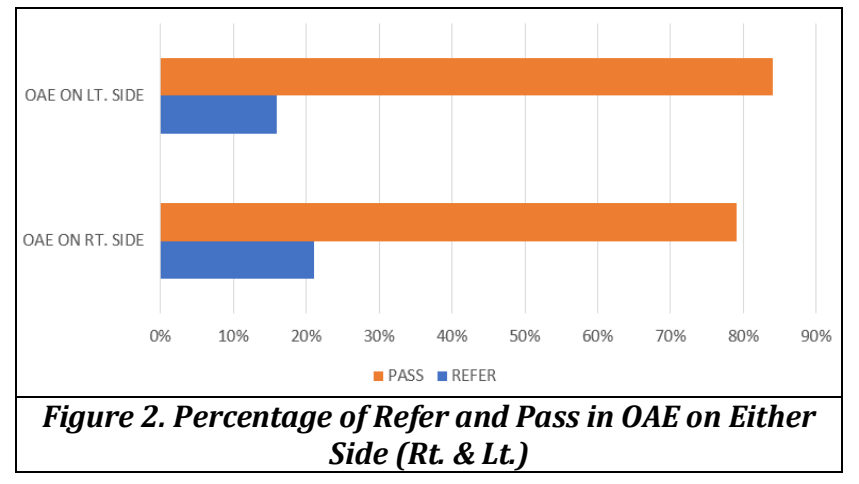

In DPOAE we have assessed in four frequencies $(2,3,4$ \& 5) KHz. Among the 39 patients who had Refer/ Absent OAE in Right side, 9 patients have affected $2 \mathrm{KHz}, 6$ patients have affected $3 \mathrm{KHz}, 6$ patients have affected $4 \mathrm{KHz}$ and 18 patients have affected $5 \mathrm{KHz}$ respectively, (Fig. 3).

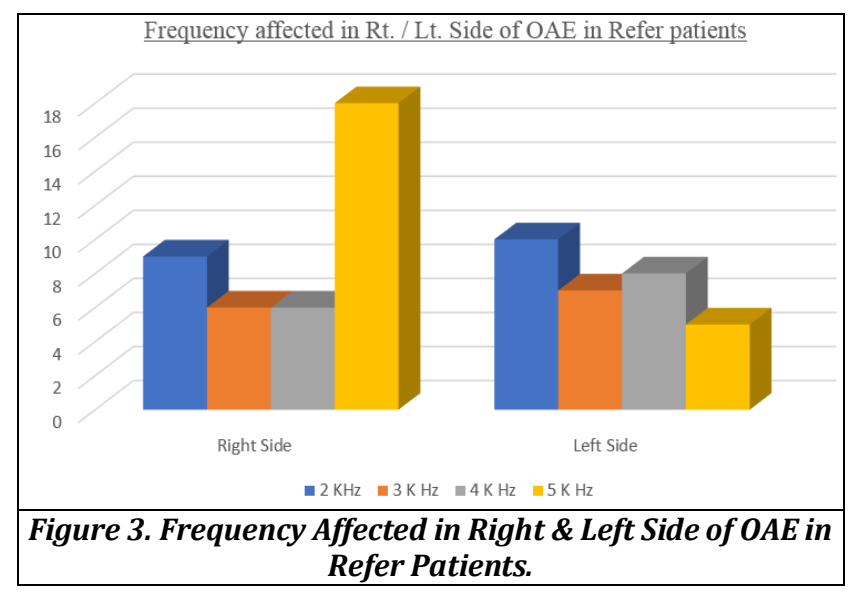

Among the 30 patients who had Refer/ Absent OAE in left side, 10 patients have affected $2 \mathrm{KHz}, 7$ patients have affected $3 \mathrm{KHz}, 8$ patients have affected $4 \mathrm{KHz}$ and 5 patients have affected $5 \mathrm{KHz}$ respectively, (Fig. 3).

\section{DISCUSSION}

Among sensory organs, Cochlea serves to two unique functions: it encodes, and processes sounds incident on the cochlea which go to the auditory cortex and also produces a characteristic sound of its own. The cochlea acts upon incoming sound with its machinery and thus changes the characteristics of vibration. Oto Acoustic emissions, or "OAEs," are by products of this mechanism. In the 1960s, von Bekesy hypothesized that the basilar membrane was tonotopically organized, and he gave a "traveling" wave or vibratory fluid motion that traversed the membrane from base to apex. According to von Bekesy, the vibratory motion on the basilar membrane reached its peak of membrane displacement at a region related to frequency of the incoming sound. This basilar membrane region associated with the characteristic frequency of a sound is determined by the acoustic properties of the sound, as well as the mass and stiffness of the membrane. As the traveling wave motion is developing and reaching its peak, the Outer hair cells (OHCs) begin to elongate and contract. Only those OHCs that are maximally stimulated become motile. And, because they are motile in only a focal region of the membrane, the displacement around this narrow cochlear region (i.e., characteristic frequency) is made larger by OHCs. This mechanical amplification of basilar membrane motion sends a clear and robust message to the brain about the acoustic input. This robust vibration pattern on the basilar membrane translates into excellent detection of Sound. This OHCmediated amplification works for sounds that are low-tomoderate in level only. The cochlear amplifier's effectiveness saturates with high level input. Without sensitive detection and exquisite frequency resolution, humans would have trouble detecting some of the critical nuances of speech; nuances that are required for adequate speech perception and discrimination.

Sahoo et al[14] conducted a similar study and among their patients $5 \%$ had Tinnitus, $4 \%$ had vertigo, $10 \%$ had ear discomfort and $8 \%$ had ear fullness. In the study by Philip P et al[15] $13.3 \%$ had sensation of blocked ear, $9.3 \%$ had burning ear, $8 \%$ had Tinnitus and $6.6 \%$ had hearing loss as complaints. In their study, in Right ear $31.3 \%$ had absent OAE in higher frequency, $8 \%$ in middle frequencies and $28 \%$ in low frequencies. In Left ear, $30.7 \%$ had absent OAE in high frequency, $8 \%$ in middle frequency and $20.7 \%$ in low frequency. Otkay MF et al [16] used PTA for evaluation and also included the data regarding frequency and duration of phone use per day and found out that detection thresholds in those who talked approximately 2 hours per day were higher than those using phone for 10-20 minutes per day. Kerekhanjanarong V. et al [17] used both Speech audiometry and Pure Tone Audiometry and found no difference between Dominant and Non-Dominant ears. They further found out that in subjects using mobile phones for more than 60 minutes per day, hearing threshold of Dominant ear was worse than Non-Dominant ears. Ramya et al [18] used PTA for assessment of hearing and their study showed significant increase in hearing thresholds at all frequencies in Right ear in test versus control group. The significant increment was not so much in the left ear in test versus control group. Panda et al [19] compared DPOAE with duration of mobile phone use per day and found out that in mobile phone using ear (Right, in majority of their cases), there was significant chances of DPOAE being associated with hours of use.

\section{Distortion Product Oto-Acoustic Emissions (DPOAE)}

In the process of amplifying and enhancing sound for efficient decoding by the brain, the cochlea creates a by-product. Just like sound that comes into the ear from an external source, the cochlear by-product produces its own physical vibration along the basilar membrane. The DPOAE is generated by the cochlea when the ear is presented with two simultaneous pure tones ( $\mathrm{f} 1$ and $\mathrm{f} 2$ ). The DPOAE travels from its generation site on the basilar membrane around the f1 and f2 frequency to the region of its own characteristic frequency. It also travels in reverse direction from its generation site, through the middle ear, and into the ear canal. The cochlear-generated distortion becomes an acoustic product once it is in the ear canal and can be measured with a sensitive microphone placed at the auditory meatus. When the DPOAE is present in the ear canal, it indicates that the mechanism generating it (i.e., the cochlear amplifier) is functional; when the DPOAE is absent, it indicates that the amplifier is nonfunctional or dysfunctional and that there is hearing loss. In this way, a distortion tone produced by the ear as a by-product and measured in the ear canal has the useful characteristic of reflecting cochlear integrity.[20] 


\section{CONCLUSION}

The study concludes that the use of mobile phones can cause damage to the Outer Hair Cells (OHCs) in the cochlea especially in the basal turn. Oto Acoustic Emission from the cochlea can reliably detect this damage and thus has proven to be an excellent diagnostic tool in early hearing loss. DPOAE defends our theory adequately that the use of mobile phones has a deteriorating effect on the vitality and function of the hair cells. Prolonged exposure to electromagnetic radiations because of constant and prolonged use of mobile phones is found to instigate this. A larger study group and a further follow up after cessation/ decreased usage of mobile phones is necessary to strengthen the hypothesis.

Mobile phones, though excellent tools for communication, have deteriorating effects owing to their electromagnetic radiations and thus their use must be limited and controlled. Like any other technology, this one too has its demerits. These effects are not limited to the ear and can be systemic. These demerits are not well known to the general public which has been using this device to the limits of addiction. They must be made aware of its harmful effects. Better public guidelines and advertisement regarding the use of mobiles must be issued in public interest by the government. This might help in decreasing the incidence of this unwanted hearing problem in patients.

$\mathrm{OAE}$ can be an excellent diagnostic and prognostic tool. Not only it helps in assessment of the hearing loss, it also guides towards the probable aetiology and the probable outcome of any treatment, if instituted. OAEs should be put to a better use by diagnosticians for a quicker and assertive management of patients with hearing loss.

\section{REFERENCES}

[1] Mobile tech03-types-of-

[2] Dimbylow PJ, Mann SM. SAR calculations in an anatomically realistic model of the head for mobile communication transceivers at $900 \mathrm{MHz}$ and $1.8 \mathrm{GHz}$. Phys Med Biol 1994;39(10):1537-53.

[3] Heynick LN, Johnston SA, Mason PA. Radio frequency electromagnetic fields: cancer, mutagenesis and genotoxicity. Bioelectromagnetics 2003;24(Suppl 6):S74-100.

[4] Frey AH. Headaches from cellular telephones: are they real and what are the implications? Environ Health Perspect 1998;106(3):101-3.

[5] Oftedal G, Wilen J, Sandstrom M, et al. Symptoms experienced in connection with mobile phone use. Occup Med (Lond) 2000;50(4):237-45.

[6] Fritze K, Sommer C, Schmitz B, et al. Effect of global system for mobile communication (GSM) microwave exposure on blood-brain barrier permeability in a rat. Acta Neuropathol (Berl) 1997;94(5):465-70.
[7] Braune S, Wrocklage C, Raczek J, et al. Resting blood pressure increase during exposure to a radiofrequency electromagnetic field. Lancet 1998;351(9119):1857-8.

[8] Borbely AA, Huber R, Graf $T$, et al. Pulsed highfrequency electromagnetic field affects human sleep and sleep electroencephalogram. Neurosci Lett 1999;275(3):207-10.

[9] Preece AW, Iwi G, Davies-Smith A, et al. Effect of a 915$\mathrm{MHz}$ simulated mobile phone signal on cognitive function in man. Int J Radiat Biol 1999;75(4):447-56.

[10] International Commission on Non-Ionizing Radiation Protection (ICNRP). Guidelines for limiting exposure to time-varying electric, magnetic and electromagnetic fields (up to $300 \mathrm{GHz}$ ). Health physics 1998;74(4):494-522.

[11] Al-Abduljawad KA. Effects of the mobile phones on the hearing function of the users. Bah Med Bull 2005.

[12] Arai N, Enomoto H, Okabe S, et al. Thirty minutes mobile phone use has no short-term adverse effects on central auditory pathways. Clin Neurophysiol 2003;114(8):1390-4.

[13] ANSI/IEEE 1992 American national standard dictionary for technologies of Electromagnetic Compatibility (EMC), Electromagnetic Pulse (EMP), and Electrostatic Discharge (ESD) (dictionary of EMC/EMP/ESD terms and definitions) accredited standards committee on electromagnetic compatibility, c63 accredited by the American national standards institute secretariat institute of electrical and electronics engineers, Inc., approved. American National Standards Institute. April 21, 1992.

[14] Sahoo GC, Sebastian H. Prevalence of sensorineural deafness in habitual mobile phone users. Indian J Otol 2011;17(3):97-100.

[15] Philip P, Bhandary SK, Aroor R, et al. The effect of mobile phone usage on hearing in adult population. Indian J Otol 2017;23(1):1-6.

[16] Oktay MF, Dasdag S. Effects of intensive and moderate cellular phone use on hearing function. Electromagn Biol Med 2006;25(1):13-21.

[17] Kerekhanjanarong V, Supiyaphun P, Naratricoon J, et al. The effect of mobile phone to audiologic system. J Med Assoc Thai 2005;88 Suppl 4:S231-4.

[18] Ramya CS, Karthiyanee K, Vinutha S. Effect of mobile phone usage on hearing threshold: a pilot study. Indian J Otol 2011;17(4):159-61.

[19] Panda NK, Jain R, Bakshi J, et al. Audiological disturbances in long-term mobile phone users. J Otolaryngol Head Neck Surg 2010;39(1):5-11.

[20] Abdala C, Visser-Dumont L. Distortion product otoacoustic emissions: a tool for hearing assessment and scientific study. Volta Rev 2001;103(4):281-302. 